\title{
Bowtie Nanoantenna with Single-Digit Nanometer Gap for Surface-Enhanced Raman Scattering (SERS)
}

\author{
Jian Zhang • Mehrdad Irannejad • Bo Cui
}

Received: 30 October 2014 / Accepted: 11 December 2014 /Published online: 23 December 2014

(C) Springer Science+Business Media New York 2014

\begin{abstract}
Sub-10-nm gaps in noble metal bowtie structures may enable strong enhancement of the near field at the gap. However, it is challenging to define such small gaps using electron beam lithography (EBL) due to the proximity effect. Here, we circumvented this problem by carrying out EBL on a thin membrane that is transparent to incident electrons and thus free from the proximity effect. Nanogaps down to $6 \mathrm{~nm}$ were obtained and employed for sensing application based on surface-enhanced Raman scattering (SERS). We achieved a high sensitivity at low concentration of the target molecule with a SERS enhancement factor of $10^{7}$.
\end{abstract}

Keywords Localized surface plasmon $\cdot$ SERS $\cdot$ EBL $\cdot$ Bowtie

\section{Introduction}

Surface-enhanced Raman scattering (SERS) [1, 2] is an ultrasensitive chemical-sensing technique capable of singlemolecule detection [3-9]. The enormous enhancement is mainly due to the localized surface plasmon resonance (LSPR) of noble metal nanoparticles (NPs) onto which the target molecules adsorb. Since the enhancement is proportional to $\left|E / E_{0}\right|^{4}$, where $E$ and $E_{0}$ are the excited and original electric near fields, strong SERS enhancement occurs at the "hotspots" with maximum electric field [9-11], such as the

J. Zhang $\cdot$ M. Irannejad $\cdot$ B. Cui $(\bowtie)$

Waterloo Institute for Nanotechnology (WIN),

University of Waterloo,

200 University Ave. W, Waterloo, Ontario N2L 3G1, Canada

e-mail: bcui@uwaterloo.ca

\section{J. Zhang}

INPAC - Institute for Nanoscale Physics and Chemistry

and Physics Department, KU Leuven,

Celestijnenlaan 200 D, B-3001 Leuven, Belgium sharp tips of the NPs [12-16], the tiny gaps between adjacent NPs [5, 17-19], or inside one NP (e.g., nanocrescent) [20, 21]. An artificial antenna such as a bowtie (two triangles facing each other) array with a nanogap is one of the most promising candidates for large enhancement since it provides simultaneously both sharp tips and tiny gaps [22-24]. Moreover, the bowtie structure fabricated using nanolithography is more reproducible and tuneable by controlling its dimension than randomly aggregated NPs or island film [3, 5, 25-27].

The near field at the gap for the bowtie structure is inversely proportional to the gap [28], and both experiment and numerical study show that a large enhancement factor occurs only for small gaps of several nanometers [29, 30]. So far, focused ion beam lithography (FIBL) is most widely utilized for the fabrication of sub-10-nm gaps due to its simple process steps [31-33]. However, this method is time-consuming for large area patterning because a tiny gap necessitates a low ion beam current of only a few $\mathrm{pA}$. In addition, Ga contamination could be a serious issue [34]. Though electron beam lithography (EBL) is faster than FIBL and free from ion contamination, it is challenging to fabricate a sub-10-nm gap due to the proximity effect originated from backscattered electrons [35-37].

To a certain degree, the proximity effect can be "diluted" by using a high-energy electron beam such as $100 \mathrm{keV}[37,38]$. However, high energy is accompanied with proportionally reduced resist sensitivity, and the "dilution" effect is insignificant when a dense bowtie array over a large surface area (larger than the range of backscattered electrons that is in the order of $50 \mu \mathrm{m}$ for $100 \mathrm{keV}$ ) is needed. A substrate with lower density and atomic number could also slightly reduce, respectively, the range and intensity of the proximity effect $[39,40]$. Lastly, the proximity effect can be compensated to a certain extent by optimizing the spatial distribution of the exposure dose using numerical computation of the electron trajectory and energy loss in the resist and substrate [41-43]. A bowtie 
with a 3-nm gap has been fabricated using such a proximity effect correction method, yet it is for an isolated bowtie structure for which the proximity effect is much smaller than a bowtie array that offers higher SERS enhancement proportional to the number of bowties within the laser beam spot.

In this work, we demonstrate the fabrication of a bowtie with a gap down to $3 \mathrm{~nm}$ by performing EBL on a thin membrane that is transparent to incident electrons and thus effectively eliminated the proximity effect. SERS study of the fabricated bowtie array was carried out using 1,2-di(4pyridyl)ethylene (BPE) as the target molecule. As expected, the enhancement was greatly dependent on the gap size of the bowtie structure.

\section{Experiment}

The bowtie structure on a thin $\mathrm{Si}_{3} \mathrm{~N}_{4}$ membrane was fabricated according to the process steps shown in Fig. 1. The nitride membrane was first fabricated using wet chemical etching with hot $\mathrm{KOH}$ solution that etches silicon anisotropically at a rate of $\sim 1 \mu \mathrm{m} / \mathrm{min}$ with negligible etching rate of the nitride film. Next, the top surface of the membrane was cleaned by $\mathrm{O}_{2}$ plasma, and a $170-\mathrm{nm}$ polymethyl methacrylate (PMMA) layer as the resist and $10 \mathrm{~nm} \mathrm{Cr}$ as the conductive charge dissipation layer were coated by spin coating and electron beam evaporation, respectively. EBL was carried out using Raith $150^{\mathrm{TWO}}$ system at $20 \mathrm{kV}$ acceleration voltage and $0.33 \mathrm{nA}$ beam current. The conductive layer was removed after exposure, and the pattern was developed using methyl isobutyl ketone (MIBK)/2-propanol (IPA) (1:3) and rinsed by fresh IPA. Finally, $1 \mathrm{~nm} \mathrm{Cr}$ as the adhesive layer and $25 \mathrm{~nm}$ Au were deposited by electron beam evaporation and lifted off by anisole. A metal film with $25-\mathrm{nm}$ thickness was selected

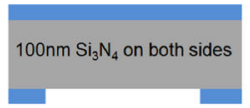

(a)

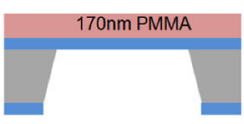

(c)

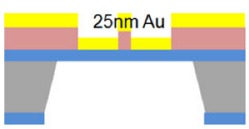

(e)

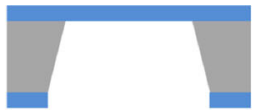

(b)

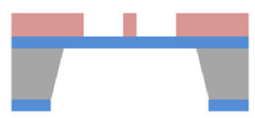

(d)

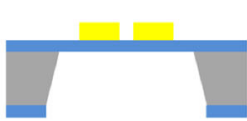

(f)
Fig. 1 Schematic of the fabrication process of bowtie nanoantenna on $\mathrm{Si}_{3} \mathrm{~N}_{4}$ membrane: a photolithography on backside $\mathrm{Si}_{3} \mathrm{~N}_{4}$ and $\mathrm{CF}_{4}$ reactive ion etching to remove the unmasked $\mathrm{Si}_{3} \mathrm{~N}_{4}$, b wet etch in $\mathrm{KOH}$ solution to form $\mathrm{Si}_{3} \mathrm{~N}_{4}$ membrane, c spin-coat $170 \mathrm{~nm}$ PMMA layer and deposit since a thicker metal film is more difficult to lift off especially when ultrasonic agitation cannot be used because of the brittle membrane. The Au film was deposited at a slow rate of $0.2 \AA /$ $\mathrm{s}$ to reduce surface roughness.

Figure 2a shows the SEM image of bowtie patterns with a 6-nm gap exposed by EBL on the bulk substrate. The gaps in the bowties are fully exposed that resulted in connected bowties. In contrast, Fig. $2 \mathrm{~b}$ shows that the bowtie nanoantenna fabricated on the $\mathrm{Si}_{3} \mathrm{~N}_{4}$ membrane with varied gap sizes in the range of 3 to $24 \mathrm{~nm}$ can be achieved. Considering the beam size of about $5 \mathrm{~nm}$, the proximity effect was eliminated completely. The narrowest gap obtained was as low as $3 \mathrm{~nm}$, though the gap size was not uniform and many connected gaps were seen in the fabricated structures. It was found that fabrication of a bowtie nanoantenna with a gap size of $6 \mathrm{~nm}$ and up is more uniform and reproducible.

The Raman spectrum was recorded by using a Bruker Senterra Raman spectrometer equipped with confocal microscope and a nitrogen-cooled multichannel CCD detector. A HeNe laser with a wavelength of $632.8 \mathrm{~nm}$ and a diode laser with a wavelength of $785 \mathrm{~nm}$ were used as the excitation sources. The laser source was focused on the sample using a $\times 100$ objective lens to obtain a spot size of $\sim 2 \mu \mathrm{m}$ diameter, and the acquisition time was set as $100 \mathrm{~s}$ for the laser wavelength of $785 \mathrm{~nm}$. For $632.8 \mathrm{~nm}$, the acquisition time was $10 \mathrm{~s}$ to minimize the high background fluorescence for the $632.8 \mathrm{~nm}$ excitation. The laser power was chosen as low as $1 \mathrm{~mW}$ to avoid burning and surface carbonization of the adsorbed organic molecules. 1,2-di(4Pyridyl)ethylene (BPE) (Sigma-Aldrich) was selected as the target molecule to be detected. A BPE solution in ethanol with a concentration of $100 \mathrm{mM}$ was diluted to a series of different concentrations in the range of $10^{-1}$ to $10^{-6} \mathrm{mM}$. The diluted solution was dropped on the bowtie nanoantenna samples and dried at room temperature before each experiment. A clean

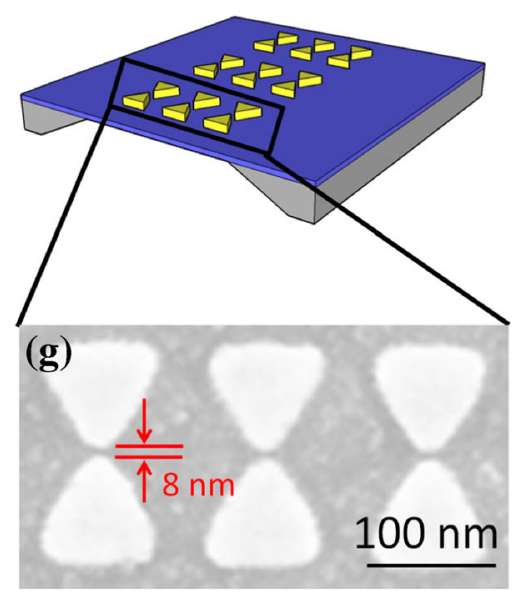

$10 \mathrm{~nm} \mathrm{Cr}$, d E-beam lithography and development, e evaporate $1 \mathrm{~nm} \mathrm{Cr}$ and $25 \mathrm{~nm} \mathrm{Au}, \mathbf{f}$ liftoff in anisole and rinse the surface, $\mathbf{g}$ illustration of the final bowtie structure on $\mathrm{Si}_{3} \mathrm{~N}_{4}$ membrane and SEM image of fabricated $\mathrm{Au}$ bowtie structure with gap size of $8 \mathrm{~nm}$ as an example 
Fig. 2 a SEM image of bowtie array exposed on bulk wafer, $\mathbf{b}$ SEM image of bowtie array exposed on $\mathrm{Si}_{3} \mathrm{~N}_{4}$ membrane

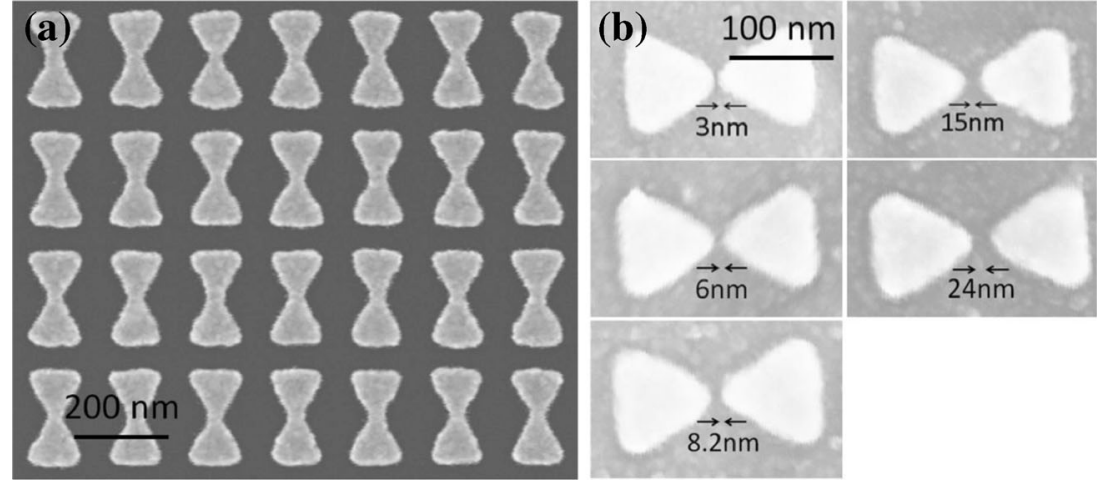

wafer coated with $10^{-1} \mathrm{mM}$ BPE was selected as the reference sample. It was found that brief $\mathrm{O}_{2}$ plasma cleaning of gold bowtie nanoantenna structures led to a more reproducible Raman measurement. Hence, the gold bowtie structure was cleaned by $\mathrm{O}_{2}$ plasma right before BPE application.

\section{Numerical Simulation}

The near-field profile in the gold bowtie nanoantenna was simulated using the 3D full wave vector finite difference time domain (FDTD) method, which is a reliable technique in solving Maxwell's equations in dispersive media. Each media was specified by a relative permittivity $\varepsilon(\omega)$. For the substrate layer, permittivity was assumed as $n^{2}$, and for the gold layer, the Lorentz-Drude model was utilized to describe its permittivity $[44,45]$. The FDTD was carried out using the Lumerical FDTD (Lumerical Solutions, Inc). The plane wave source, bowtie structure, and monitors were co-planar with boundary conditions that made them effectively infinite. In this study, a plane wave of linearly polarized light along the $Y$-axis $\left(\lambda_{\text {center }}\right.$ $=680 \mathrm{~nm}$, time offset of $0.8 \times 10^{-14} \mathrm{~s}$ and half width of $0.1 \times$ $10^{-14} \mathrm{~s}$ ) which propagates along the $Z$-axis was used. The simulation background was taken as air $(n=1.0)$. The gap in the bowtie structure was varied in the range of 6 to $24 \mathrm{~nm}$. A metal film with a $25-\mathrm{nm}$ thickness was selected corresponding to the fabricated device. The simulation cell was considered as $550 \times 550 \times 400 \mathrm{~nm}^{3}$, and the periodic boundary condition (PBC) was used in the $X$ - and $Y$-directions. An anisotropic perfect matching layer (APML) was used in the $Z$-direction as absorbing boundary condition. The calculation grid resolution (grid point-to-point distance) was as high as 1 to $5 \mathrm{~nm}$ (at different structures with different gap sizes) in the simulation cell. The calculation time was set as $100 \mathrm{fs}$, with a calculation step size of $0.00834 \mathrm{fs}$ for a total of about 12,000 time steps per simulation. The transmission spectra were calculated using an $X-Y$ monitor at $150 \mathrm{~nm}$ away from the bowtie nanoantenna surface.

\section{Results and Discussion}

Numerical Analysis

For a metallic gap with an ultranarrow gap size, FDTD simulation may not be accurate because both plasmonic coupling and quantum tunnelling may happen. In our work, FDTD simulation is still considered since the electron interaction is within classical regime (gap size $>1 \mathrm{~nm}$ ) $[46,47]$. FDTD in Fig. 3a shows the FDTD calculated electric field profile, $|E|^{2}$, of the gold bowtie with a gap size of $6 \mathrm{~nm}$ at an excitation wavelength of $785 \mathrm{~nm}$. As can be seen from this figure, the electric field was highly confined in the gap area. It was also found that on changing the excitation wavelength from 785 to $632.8 \mathrm{~nm}$, the electric field confinement in the gap area was eliminated and more electric field propagation was observed on the triangle sides of the bowtie structures as it is clear from Fig. 3b. This indicates that $785 \mathrm{~nm}$ is close to the surface plasmon resonance wavelength of the bowtie. Correspondingly, the following Raman measurement in Fig. $4 a-d$ shows the different enhancement using the excitation wavelengths of 785 and $632.8 \mathrm{~nm}$. Also, a longer wavelength is preferred to reduce the background fluorescence effects during irradiation of organic molecules.

\section{Surface-Enhanced Raman Spectroscopy}

Noble metals such as gold and silver are usually employed in SERS applications because of their inert activity, adsorbility to the amino/thiol group, high surface plasmon resonance amplitude, and low loss [48]. It is more interesting to use gold since its surface is more stable than silver in atmospheric conditions.

A series of bowtie nanoantenna structures with different periods in the range of 550 to $1000 \mathrm{~nm}$ with step of $150 \mathrm{~nm}$ were fabricated to study the effect of bowtie density on the SERS signal enhancement. The effects of varying structural periodicity on the Raman signal intensity of the gold bowtie nanoantenna of gap size of $6 \mathrm{~nm}$ is shown in Fig. 4a, b. As 


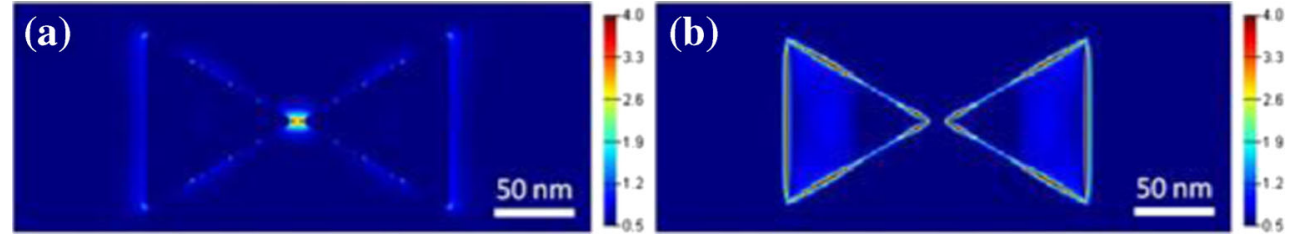

Fig. 3 FDTD calculated electric field intensity, $|E|^{2}$, on gold bowtie structure with $6 \mathrm{~nm}$ gap illuminated by excitation wavelength of $\mathbf{a} 785 \mathrm{~nm}$ and $\mathbf{b}$ $632.8 \mathrm{~nm}$

it is clear on reducing the periodicity from 1000 to $550 \mathrm{~nm}$, the recoded Raman intensities at the wavenumber of $1610 \mathrm{~cm}^{-1}(\mathrm{C}=\mathrm{N}$ bond) were increased nearly linearly with the increasing bowtie density and a bowtie array of $1000 \mathrm{~nm}$ period and $6 \mathrm{~nm}$ gap could still support a detectable Raman signal.

The effects of varying the gap size and excitation wavelength on the recorded SERS signal are compared in Fig. 4cd. For an excitation wavelength of $785 \mathrm{~nm}$, the Raman intensity of BPE molecules was increased by nearly two orders of magnitude on reducing the gap size from 24 to $6 \mathrm{~nm}$. However, in case of using the excitation wavelength of $632.8 \mathrm{~nm}$, there was no significant change in the Raman intensity of BPE molecules in the bowtie nanoantenna structures with different gap sizes, which is mainly due to the absence of a hotspot at this excitation wavelength.
The enhancement factor (EF) is one of the most important parameters in SERS application, and it is given by

$\mathrm{EF}=\frac{I_{\text {SERS }} / N_{\text {SERS }}}{I_{\text {Ref }} / N_{\text {Ref }}}$

where $I_{\text {SERS }}$ and $I_{\text {Ref }}$ are the measured Raman intensities of the SERS and the reference samples, and $N_{\text {SERS }}$ and $N_{\text {Ref }}$ are the numbers of molecules on the surface of the bowtie and the reference sample. The ratio of $N_{\mathrm{SERS}} / N_{\mathrm{Ref}}$ can be estimated as

$\frac{N_{\text {SERS }}}{N_{\text {Ref }}} \approx \frac{M_{\text {SERS }} \cdot \text { Volume of liquid on SERS }}{M_{\text {Ref }} \cdot \text { Volume of liquid on reference }}$

$$
=\frac{M_{\mathrm{SERS}} \cdot A_{\mathrm{Bowtie}} \cdot h}{M_{\mathrm{Ref}} \cdot A_{\mathrm{Ref}} \cdot h}=\frac{M_{\mathrm{SERS}} \cdot A_{\mathrm{Bowtie}}}{M_{\mathrm{Ref}} \cdot A_{\mathrm{Ref}}}
$$

Fig. 4 a SERS spectrum of $0.1 \mathrm{mM}$ BPE from bowtie array having $6 \mathrm{~nm}$ gap and different periods in the range of 550 to $1000 \mathrm{~nm}$, with laser excitation wavelength of $785 \mathrm{~nm}$. The spectrum was acquired with an integration time of $100 \mathrm{~s}$. b The relationship between Raman intensity at $1610 \mathrm{~cm}^{-1}(\mathrm{C}=\mathrm{N}$ bond) and the bowtie density, derived from a. c, d Raman spectrum for bowtie structure having period of $550 \mathrm{~nm}$ and different gap sizes, with laser excitation wavelength of 785 and $632.8 \mathrm{~nm}$, respectively. Both spectrum were acquired with an integration time of $10 \mathrm{~s}$ (a)

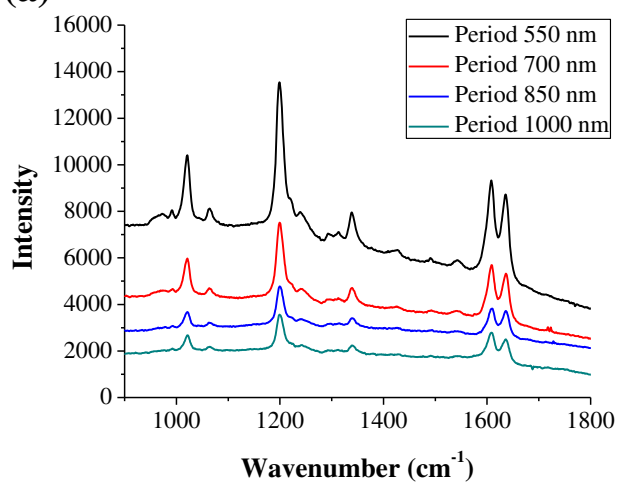

(c)

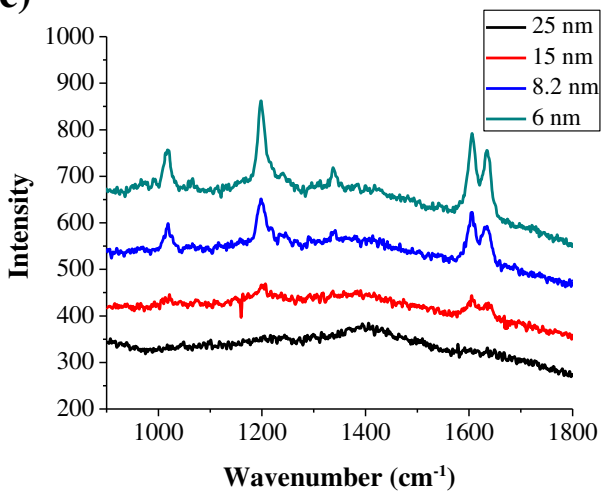

(b)

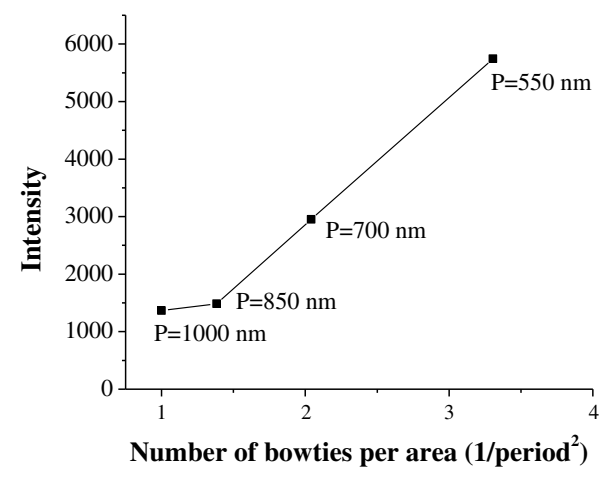

(d)

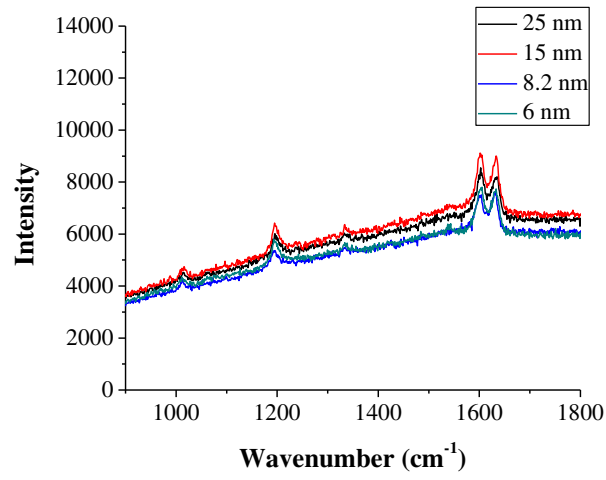


Fig. 5 Recorded SERS spectra of BPE with different concentrations in the range of 0.1 to $10^{-6} \mathrm{mM}$ from bowtie nanoantenna structure with gap sizes of a $6 \mathrm{~nm}$ and b $24 \mathrm{~nm}$. c, d The relationship between the recorded Raman intensity of $1610 \mathrm{~cm}^{-1}(\mathrm{C}=\mathrm{N}$ bond) and the BPE concentration, derived from the $\mathbf{a}$ and $\mathbf{b}$ results, respectively. The array period is $550 \mathrm{~nm}$, and the laser excitation wavelength is $785 \mathrm{~nm}$
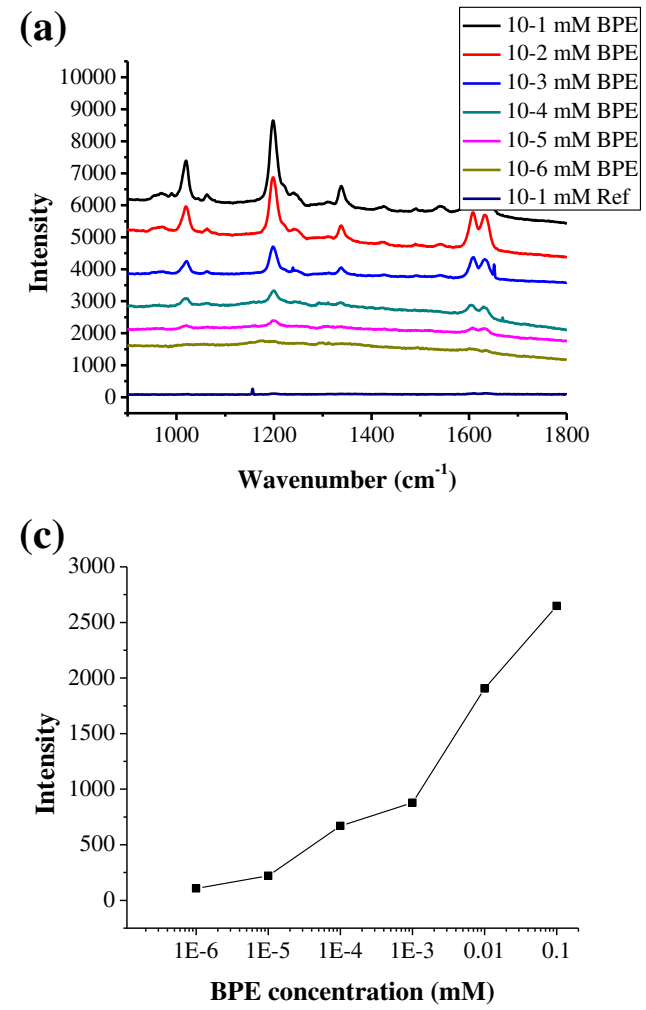

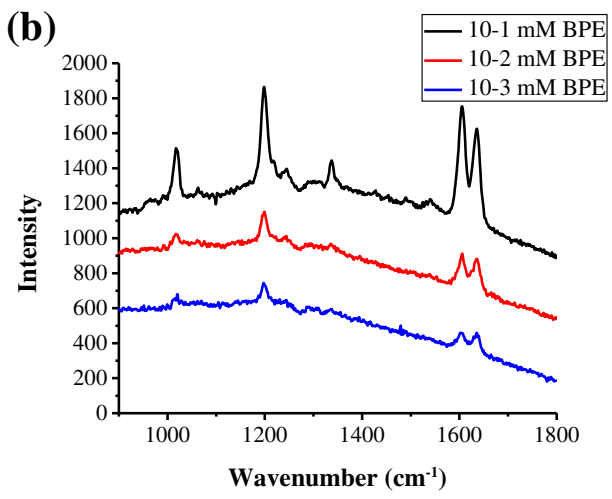

(d)

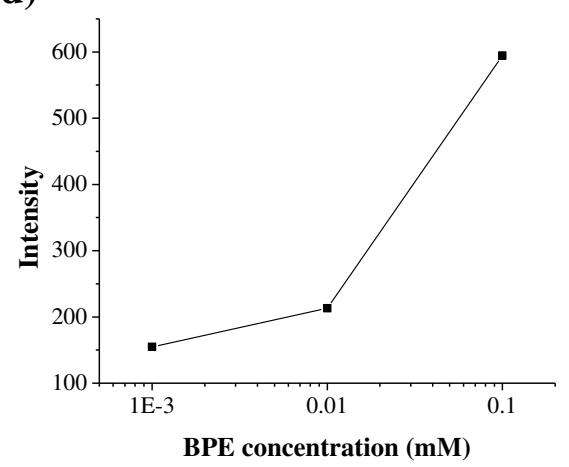

where $M_{\mathrm{SERS}}$ and $M_{\mathrm{Ref}}$ are the molecular concentrations of the SERS and reference samples, $A_{\text {Bowtie }}$ is the area of the bowties, and $A_{\text {Ref }}$ is the exposure area of the laser beam on the reference sample, which is equal to the beam area. Importantly, since in the bowtie nanoantenna the hotspot only exists within the gap area, the actual effective area is much smaller than the whole bowtie area and hence the corresponding EF at the gap is much larger than the calculated one.

Figure $5 \mathrm{a}, \mathrm{b}$ shows the Raman spectra of the bowtie nanoantenna with gap sizes of 6 and $24 \mathrm{~nm}$ at different BPE concentrations in the range of $10^{-1}$ to $10^{-6} \mathrm{mM}$ based on the excitation wavelength of $785 \mathrm{~nm}$. It was found that the bowtie nanoantenna with a gap size of $6 \mathrm{~nm}$ achieved an EF of $10^{7}$ at a BPE concentration of $10^{-5} \mathrm{mM}$. Whereas on using a nanoantenna with a gap size of $24 \mathrm{~nm}$, the highest sensitivity was recorded at a BPE concentration of $10^{-3} \mathrm{mM}$ with an EF of $10^{5}$ as shown in Fig. 5b. This confirms that the hotspot existed only at the nanogap between facing tips of the bowtie structure and the amplitude of the local electric field was significantly increased by reducing the gap size, which is in good agreement with numerical results.

\section{Conclusion}

Here, we demonstrated electron beam lithography with a negligible proximity effect by exposure on top of a thin
$\mathrm{Si}_{3} \mathrm{~N}_{4}$ membrane that is transparent to incident electrons. We obtained a bowtie array structure with a gap size down to $3 \mathrm{~nm}$. The dependence of the excitation wavelength and structural parameters including gap size and array periodicity on the SERS performance was studied experimentally and numerically. The Raman intensity showed a strong dependence on the size of the gap where exists the "hotspot" at resonance. On using an excitation wavelength of $785 \mathrm{~nm}$, the hotspot was localized at the gap area leading to a strong SERS enhancement. However, for an excitation wavelength of $632.8 \mathrm{~nm}$, the spots with a relatively high near field were located along the triangle edges (rather than within the gap) with an intensity far lower than that at the gap for the case of 785-nm excitation, which resulted in a low Raman enhancement.

Acknowledgments This work was carried out using the nanofabrication facility at Quantum NanoFab funded by the Canada Foundation for Innovation, the Ontario Ministry of Research \& Innovation, and Industry Canada.

\section{References}

1. Haynes CL, Yonzon CR, Zhang X, Van Duyne RP (2005) Surfaceenhanced Raman sensors: early history and the development of sensors for quantitative biowarfare agent and glucose detection. $\mathrm{J}$ Raman Spectrosc 36(6-7):471-484 
2. Tian Z-Q, Ren B (2004) Adsorption and reaction at electrochemical interfaces as probed by surface-enhanced Raman spectroscopy. Annu Rev Phys Chem 55:197-229

3. Kneipp K, Wang Y, Kneipp H, Perelman LT, Itzkan I, Dasari RR, Feld MS (1997) Single molecule detection using surface-enhanced Raman scattering (SERS). Phys Rev Lett 78(9):1667-1670

4. Le Ru EC, Meyer M, Etchegoin PG (2006) Proof of single-molecule sensitivity in surface enhanced Raman scattering (SERS) by means of a two-analyte technique. J Phys Chem B 110(4):1944-1948

5. Michaels AM, Jiang J, Brus L (2000) Ag nanocrystal junctions as the site for surface-enhanced Raman scattering of single rhodamine $6 \mathrm{G}$ molecules. J Phys Chem B 104(50):11965-11971

6. Xu HX, Bjerneld EJ, Kall M, Borjesson L (1999) Spectroscopy of single hemoglobin molecules by surface enhanced Raman scattering. Phys Rev Lett 83(21):4357-4360

7. Dieringer JA, Lettan RB, Scheidt KA, Van Duyne RP (2007) A frequency domain existence proof of single-molecule surface-enhanced Raman spectroscopy. J Am Chem Soc 129(51):16249-16256

8. Qian XM, Nie SM (2008) Single-molecule and single-nanoparticle SERS: from fundamental mechanisms to biomedical applications. Chem Soc Rev 37:912-920

9. Camden JP, Dieringer JA, Wang YM, Masiello DJ, Marks LD, Schatz GC, Van Duyne RP (2008) Probing the structure of singlemolecule surface-enhanced Raman scattering hot spots. J Am Chem Soc 130(38):12616-112617

10. Moskovits M (2011) Imaging spot the hotspot. Nature 469:307-308

11. Cang H, Labno A, Lu CG, Yin XB, Liu M, Gladden C, Liu YM, Zhang X (2001) Probing the electromagnetic field of a 15-nanometre hotspot by single molecule imaging. Nature 469:385-388

12. Hartschuh A, Anderson N, Novotny L (2003) Near-field Raman spectroscopy using a sharp metal tip. J Microsc (Oxf U K) 210: 234-240

13. Pazos-Perez N, Barbosa S, Rodriguez-Lorenzo L, Aldeanueva-Potel P, Perez-Juste J, Pastoriza-Santos I, Alvarez-Puebla RA, Liz-Marzan LM (2010) Growth of sharp tips on gold nanowires leads to increased surface-enhanced Raman scattering activity. J Phys Chem Lett 1(1): 24-27

14. Aldeanueva-Potel P, Carbo-Argibay E, Pazos-Perez N, Barbosa S, Pastoriza-Santos I, Alvarez-Puebla RA, Liz-Marzan LM (2012) Spiked gold beads as substrates for single-particle SERS. Chemphyschem 13(10):2561-2565

15. Song HM, Deng L, Khashab NM (2013) Intracellular surfaceenhanced Raman scattering (SERS) with thermally stable gold nanoflowers grown from Pt and Pd seeds. Nanoscale 5(10):43214329

16. Cui B, Clime L, Li K, Veres T (2008) Fabrication of large area nanoprism arrays and their application for surface enhanced Raman spectroscopy. Nanotechnology 19(14):145302

17. Hinde RJ, Sepaniak MJ, Compton RN, Nordling J, Lavrik N (2001) Surface-enhanced resonance Raman scattering of adsorbates under liquid nitrogen. Chem Phys Lett 339(3-4):167-173

18. Speed JD, Johnson RP, Hugall JT, Lal NN, Bartlett PN, Baumberg JJ, Russell AE (2011) SERS from molecules bridging the gap of particlein-cavity structures. Chem Commun 47:6335-6337

19. Merk V, Kneipp J, Leosson K (2013) Gap size reduction and increased SERS enhancement in lithographically patterned nanoparticle arrays by templated growth. Adv Opt Mater 1(4):313-318

20. Li K, Clime L, Cui B, Veres T (2008) Surface enhanced Raman scattering on long-range ordered noble-metal nanocrescent arrays. Nanotechnology 19(14):145305

21. Cui B, Veres T (2007) Fabrication of metal nanoring array by nanoimprint lithography (NIL) and reactive ion etching. Microelectron Eng 84(5-8): 1544-1547

22. Fromm DP, Sundaramurthy A, Schuck PJ, Kino G, Moerner WE (2004) Gap-dependent optical coupling of single "bowtie" nanoantennas resonant in the visible. Nano Lett 4(5):957-961
23. Kinkhabwala A, Yu ZF, Fan SH, Avlasevich Y, Mullen K, Moerner WE (2009) Large single-molecule fluorescence enhancements produced by a bowtie nanoantenna. Nat Photon 3:654-657

24. Fromm DP, Sundaramurthy A, Kinkhabwala A, Schuck PJ, Kino GS, Moerner WE (2006) Exploring the chemical enhancement for surface-enhanced Raman scattering with Au bowtie nanoantennas. J Chem Phys 124(6):061101

25. Zhang XY, Yonzon CR, Van Duyne RP (2006) Nanosphere lithography fabricated plasmonic materials and their applications. J Mater Res 21(5):1083-1092

26. Nie SM, Emery SR (1997) Probing single molecules and single nanoparticles by surface-enhanced Raman scattering. Science 275 (5303):1102-1106

27. Geissler M, Li K, Cui B, Clime L, Veres T (2009) Plastic substrates for surface-enhanced Raman scattering. J Phys Chem C 113(40): $17296-17300$

28. Kwon SH (2013) Plasmonic ruler with angstrom distance resolution based on double metal blocks. IEEE Photon Technol Lett 25(16): $1619-1622$

29. Qin L, Zou S, Xue C, Atkinson A, Schatz GC, Mirkin CA (2006) Designing, fabricating, and imaging Raman hot spots. Proc Natl Acad Sci U S A 103(36):13300-13303

30. Hu M, Ou FS, Wu W, Naumov I, Li XM, Bratkovsky AM, Williams RS, Li ZY (2010) Gold nanofingers for molecule trapping and detection. J Am Chem Soc 132(37):12820-12822

31. Hatab NA, Hsueh CH, Gaddis AL, Retterer ST, Li JH, Eres G, Zhang ZY, Gu BH (2010) Free-standing optical gold bowtie nanoantenna with variable gap size for enhanced Raman spectroscopy. Nano Lett 10(12):4952-4955

32. Dhawan A, Gerhold M, Vo-Dinh T (2007) Theoretical simulation and focused ion beam fabrication of gold nanostructures for surfaceenhanced Raman scattering (SERS). Nanobiotechnol 3(3-4):164 171

33. Lin YY, Liao JD, Ju YH, Chang CW, Shiau AL (2011) Focused ion beam-fabricated $\mathrm{Au}$ micro/nanostructures used as a surface enhanced Raman scattering-active substrate for trace detection of molecules and influenza virus. Nanotechnology 22(18): 185308

34. Melli M, Polyakov A, Gargas D, Huynh C, Scipioni L, Bao W, Ogletree DF, Schuck PJ, Cabrini S, Weber-Bargioni A (2013) Reaching the theoretical resonance quality factor limit in coaxial plasmonic nanoresonators fabricated by helium ion lithography. Nano Lett 13(6):2687-2691

35. Chang THP (1975) Proximity effect in electron-beam lithography. J Vac Sci Technol 12:1271-1275

36. Lee SY, Anbumony K (2006) Analysis of three-dimensional proximity effect in electron-beam lithography. Microelectron Eng 83(2): 336-344

37. Webster MN, Verbruggen AH, Romijn J, Jos HFF, Moors PMA, Radelaar S (1992) Proximity effect in high-voltage electron-beam lithography on Ti/Pt/Au metallization. Microelectron Eng 17(1-4): 29-32

38. IIs P, Michel M, Forchel A, Gyuro I, Speier P, Zielinski E (1994) Fabrication of ultrasmall $\mathrm{InGaAs} / \mathrm{InP}$ nanostructures by high-voltage electron-beam lithography and wet chemical etching. Nato Adv Sci Inst Serv 264:77-80

39. Zhang J, Fouad M, Yavuz M, Cui B (2001) Charging effect reduction in electron beam lithography with nA beam current. Microelectron Eng 88(8):2196-2199

40. Czaplewski DA, Holt MV, Ocola LE (2013) The range and intensity of backscattered electrons for use in the creation of high fidelity electron beam lithography patterns. Nanotechnology 24 (30):305302

41. Frye RC, Rietman EA, Cummings KD (1990) Computation of proximity effect corrections in electron-beam lithography by a neural network. IEEE International Conference on Systems, Man, and Cybernetics: A7-A14 
42. Seo E, Kim O (2000) Dose and shape modification proximity effect correction for forward-scattering range scale features in electron beam lithography. Microprocesses and Nanotechnology, Digest of Papers: $158-159$

43. Manheller M, Trellenkamp S, Waser R, Karthauser S (2012) Reliable fabrication of $3 \mathrm{~nm}$ gaps between nanoelectrodes by electron-beam lithography. Nanotechnology 23(12):125302

44. Vial A, Laroche T (2007) Description of dispersion properties of metals by means of the critical points model and application to the study of resonant structures using the FDTD method. J Phys D Appl Phys 40(22):7152
45. Rakic AD, Djurisic AB, Elazar JM, Majewski ML (1998) Optical properties of metallic films for vertical-cavity optoelectronic devices. Appl Opt 37(22):5271-5283

46. Zuloaga J, Prodan E, Nordlander P (2009) Quantum description of the plasmon resonances of a nanoparticle dimer. Nano Lett 9(2):887891

47. Savage JK, Hawkeye MM, Esteban R, Borisov GA, Aizpurua J, Baumberg JJ (2012) Revealing the quantum regime in tunnelling plasmonics. Nature 491:574-577

48. Astilean S (2007) Noble-metal nanostructures for controlling surface plasmons and sensing molecules. Radiat Phys Chem 76(3):436-439 